ARTICLE

\title{
Metallic tin quantum sheets confined in graphene toward high-efficiency carbon dioxide electroreduction
}

Fengcai Lei ${ }^{1, \star}$, Wei Liu ${ }^{2, \star}$, Yongfu Sun ${ }^{1,3}$, Jiaqi $X u^{1}$, Katong Liu¹, Liang Liang ${ }^{1}$, Tao Yao ${ }^{2,3}$, Bicai Pan ${ }^{1}$, Shiqiang Wei ${ }^{2,3}$ $\& Y i X_{i} e^{1,3}$

Ultrathin metal layers can be highly active carbon dioxide electroreduction catalysts, but may also be prone to oxidation. Here we construct a model of graphene confined ultrathin layers of highly reactive metals, taking the synthetic highly reactive tin quantum sheets confined in graphene as an example. The higher electrochemical active area ensures 9 times larger carbon dioxide adsorption capacity relative to bulk tin, while the highly-conductive graphene favours rate-determining electron transfer from carbon dioxide to its radical anion. The lowered tin-tin coordination numbers, revealed by $\mathrm{X}$-ray absorption fine structure spectroscopy, enable tin quantum sheets confined in graphene to efficiently stabilize the carbon dioxide radical anion, verified by 0.13 volts lowered potential of hydroxyl ion adsorption compared with bulk tin. Hence, the tin quantum sheets confined in graphene show enhanced electrocatalytic activity and stability. This work may provide a promising lead for designing efficient and robust catalysts for electrolytic fuel synthesis.

\footnotetext{
${ }^{1}$ Hefei National Laboratory for Physical Sciences at Microscale, Collaborative Innovation Center of Chemistry for Energy Materials, University of Science \& Technology of China, Hefei, Anhui 230026, China. ${ }^{2}$ National Synchrotron Radiation Laboratory, University of Science and Technology of China Hefei, Anhui 230029, China. ${ }^{3}$ Hefei Science Center of CAS, Hefei, Anhui 230061, China. * These authors contributed equally to this work. Correspondence and requests for materials should be addressed to Y.S (email: yfsun@ustc.edu.cn) or to T.Y. (email: yaot@ustc.edu.cn) or to Y.X. (email: yxie@ustc.edu.cn).
} 
$\mathrm{T}$ he excessive utilization of fossil fuels provoked the increasing energy crisis and the worsening global climate, which triggered tremendous attention at $\mathrm{CO}_{2}$ capture, storage and utilization ${ }^{1-3}$. To address these issues, electrocatalytic reduction of $\mathrm{CO}_{2}$ into hydrocarbon fuels is considered as a promising strategy ${ }^{1-3}$, among which metal electrodes, benefiting from high electronic conductivity, show considerable catalytic activities toward $\mathrm{CO}_{2}$ electroreduction ${ }^{4-6}$. Nevertheless, the practical applications of these metal electrodes are greatly impeded by the very low energetic efficiencies, which could be primarily ascribed to their extremely low amount of catalytically active sites ${ }^{7}$. Very recently, our study demonstrates that the ultrathin layers of metallic Co exhibit roughly 26 times higher $\mathrm{CO}_{2}$ reduction activity relative to bulk Co (ref. 2), profiting from the former's ultralarge fraction of metal atoms exposed on the surface. In spite of such a significant advantage, not all metals could be easily fabricated into ultrathin layers for substantially promoting $\mathrm{CO}_{2}$ reduction performances. For some metals with higher chemical reactivity, while exposing ultrahigh amount of surface atoms, they tend to be very unstable at ambient conditions and will be oxidized in a non-controlled manner ${ }^{1,5,6,8}$, which would lead to the loss of electronic conductivity and consequently cause a rapid decay in the cyclability. As such, developing a new strategy for stabilizing the ultrathin layers of highly reactive metals is highly imperative for both fundamental research and practical application in $\mathrm{CO}_{2}$ electroreduction.

Motivated by the above consideration, we construct a material model of ultrathin metal layers confined in graphene, in which the protection of graphene could avoid the oxidation of the highly reactive metals at ambient atmosphere (Fig. 1). The metal ultrathin layers could not only serve as a 'spacer' to favour electrolyte diffusion into the matrix of graphene ${ }^{9}$, but also afford abundant surface atoms to act as the active sites for efficient $\mathrm{CO}_{2}$ adsorption ${ }^{10,11}$, hence providing the prerequisite to involve the following reduction reactions. That is to say, in our designed sandwich-like structure, there is enough space between the graphene layers and hence the electrolyte can freely diffuse into/out the deep matrix of graphene. In this case, $\mathrm{CO}_{2}$ could diffuse into the graphene layers through dissolving in the electrolyte and simultaneously the products could also be transported out of the graphene layers by virtue of the freely flowing electrolyte. Moreover, the highly-conductive graphene endures fast electron penetration to the metal ultrathin layers ${ }^{12}$, which hence favours the rate-determining electron transfer from $\mathrm{CO}_{2}$ to $\mathrm{CO}_{2}^{-}$intermediate. In addition, as revealed by X-ray absorption fine structure spectroscopy (XAFS) in our previously systematic studies ${ }^{13,14}$, the exposed surface metal atoms would possess lowered coordination number compared with interior atoms, in which the low-coordinated metal atoms with many dangling bonds could help to stabilize the $\mathrm{CO}_{2}^{-}$intermediate, hence lowering the overall activation energy barrier and remarkably improving the catalytic activity. Furthermore, the intimate contact between ultrathin metal layers and graphene in a sandwich-like fashion could ensure excellent long-term stability.

\section{Results}

Characterizations of Sn quantum sheets confined in graphene. Of note, the low cost and environmentally benign Sn is not only one of the most active metals, but also possesses selective reduction of $\mathrm{CO}_{2}$ to formate ${ }^{5,8,15}$, which enables it to be a highly reactive metal candidate for investigating the corresponding $\mathrm{CO}_{2}$ reduction performances. Therefore, it is highly warranted for controllably synthesizing Sn ultrathin layers in the confined space of graphene. Herein, metallic Sn quantum sheets confined in graphene were first successfully synthesized through a spatially confined reduction strategy (Fig. 2a). The as-prepared ultrathin $\mathrm{SnO}_{2}$ layers ${ }^{16}$ were initially coated with amorphous carbon through a hydrothermal method. Hence, after subsequent temperature-programmed calcination for the carbon-coated ultrathin $\mathrm{SnO}_{2}$ layers, the amorphous carbon turned into graphene, while the ultrathin $\mathrm{SnO}_{2}$ layers simultaneously reduced into monodispersed Sn quantum sheets in the confined space of graphene, benefiting from the short calcination time and rapid subsequent cooling in an inert atmosphere of Ar gas.

With regard to the precise characterization of ultrathin $\mathrm{Sn}$ layers, it encounters many great difficulties since the lack of long-range order in the third dimensionality causes the typical X-ray diffraction technique unable to identify the spatial atomic distribution, while the XPS spectrum is only a surface-sensitive spectroscopic technique ${ }^{7}$. In this regard, high-resolution transmission electron microscopy (HRTEM) image and synchrotron radiation XAFS spectroscopy were utilized to characterize the phase and atomic structure of the synthetic Sn quantum sheets. Transmission electron microscopy (TEM) image in Fig. 2b shows the extremely thin sheet-like morphology of graphene, in which the Raman bands at around $1,352,1,593$ and $2,700 \mathrm{~cm}^{-1}$ demonstrated the formation of graphene (Fig. $2 \mathrm{~g})^{17}$. In addition, HRTEM image in Fig. $2 \mathrm{c}$ also shows that abundant two-dimensional (2D) sheets nearly monodispersed on the graphene, while the interplanar spacing of 0.299 and $0.277 \mathrm{~nm}$ as well as the corresponding dihedral angle of $61.4^{\circ}$ could index them to be tetragonal Sn. It should be noted that the Sn quantum sheets were encapsulated in graphene rather than exposed on the outer surface (Fig. 2f), which could be further implied by the thermogravimetric (TG) result in Fig. $2 \mathrm{~h}$. The TG analysis in air showed a rapid weight loss between 390 and $570{ }^{\circ} \mathrm{C}$, corresponding to the decomposition of gaphene, and a weight increase at above $570^{\circ} \mathrm{C}$, owing to the oxidation of

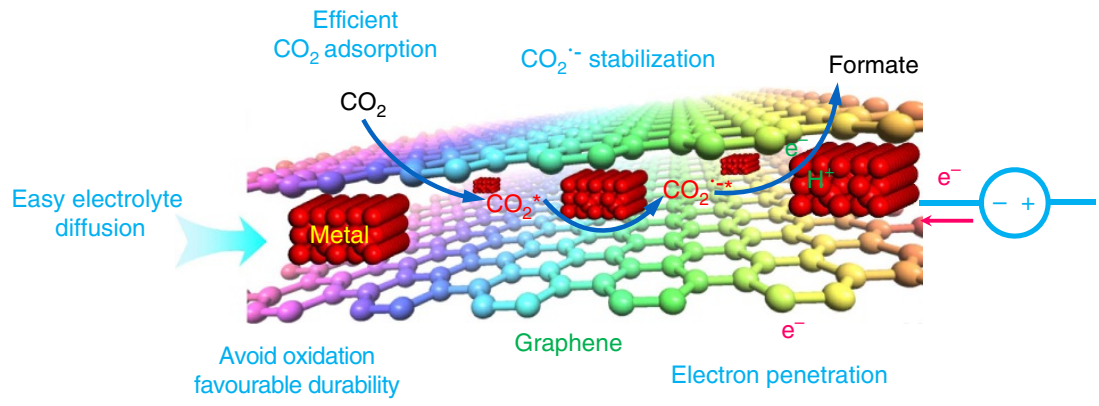

Figure 1 | Scheme. Schematic illustration depicts the several advantages of ultrathin metal layers confined in graphene for $\mathrm{CO}_{2}$ electroreduction into hydrocarbon fuels. 
a
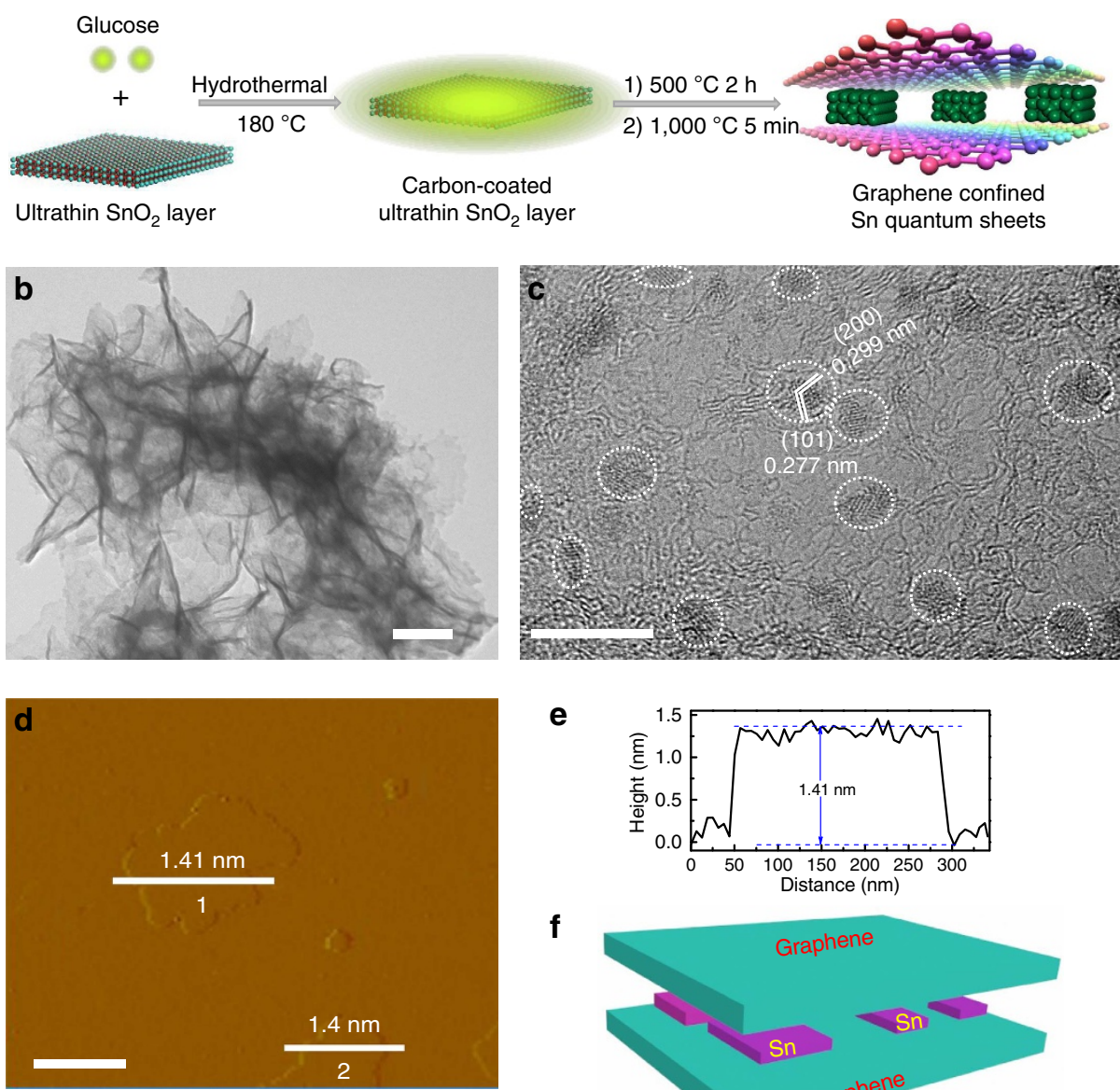

e

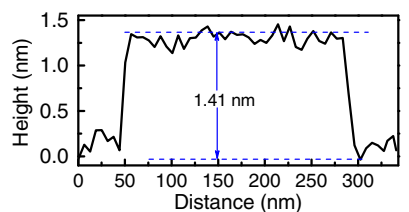

f

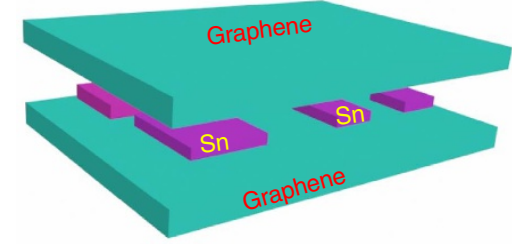

g

h
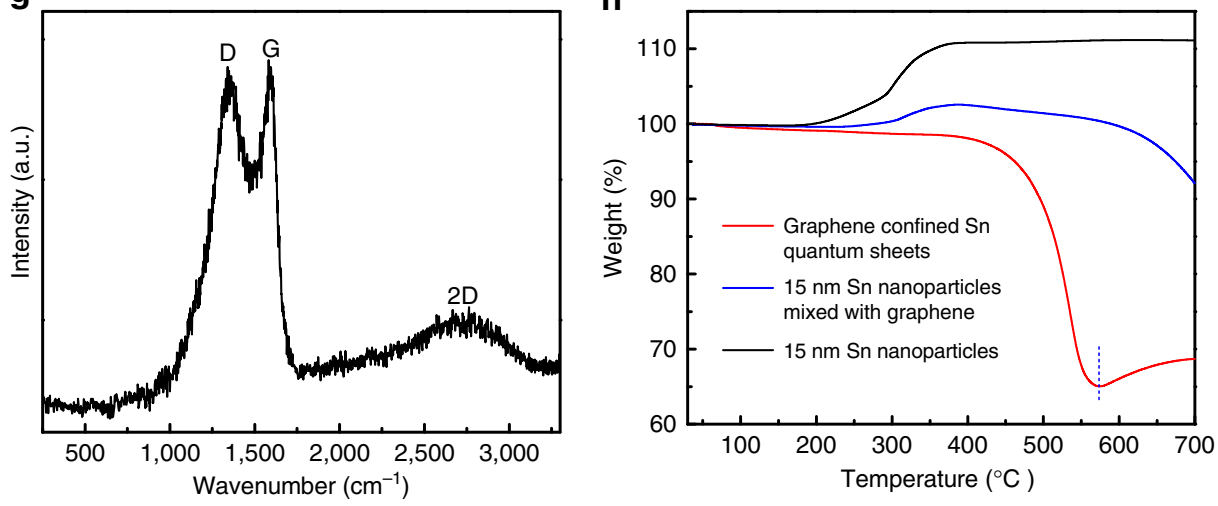

Figure 2 | Formation process and characterizations of the Sn quantum sheets confined in graphene. (a) Scheme illustration for the formation of Sn quantum sheets confined in graphene, (b) TEM image, (c) HRTEM image, (d-f) AFM image, the corresponding height profile and scheme illustration and (g) micro-Raman spectrum of the Sn quantum sheets confined in graphene. (h) TG analysis of Sn quantum sheets confined in graphene, $15 \mathrm{~nm}$ Sn nanoparticles and $15 \mathrm{~nm}$ Sn nanoparticles mixed with graphene. The scale bars in (b-d) are 100, 10 and $200 \mathrm{~nm}$, respectively. The inset circles in (c) denote the presence of Sn quantum sheets.

metallic Sn, which indicated that the protection of graphene enabled the Sn quantum sheets to be stable in air up to $570^{\circ} \mathrm{C}$. Contrastingly, both the synthetic $15 \mathrm{~nm} \mathrm{Sn} \mathrm{nanoparticles} \mathrm{and}$ their mixture with graphene showed a weight increase at above ca. $200^{\circ} \mathrm{C}$ (see Methods for details), indicating that the metallic $\mathrm{Sn}$ is easily oxidized without the protection of graphene interlayer. As a result, the presence of graphene interlayer not only avoided the oxidation of metallic $\mathrm{Sn}$, but also prevented the aggregation of quantum sized Sn sheets. Moreover, as revealed by the atomic force microscopic image and the corresponding height profiles in Fig. 2d-e, the sandwich-like structure possessed an average thickness of ca. $1.4 \mathrm{~nm}$, which indicated that the thickness of either $\mathrm{Sn}$ or graphene layer was much less than $1.4 \mathrm{~nm}$. Accordingly, the above results demonstrated the formation of monodisperse Sn quantum sheets confined in graphene.

To further identify the phase and unravel the local atomic arrangements of the synthetic Sn quantum sheets, synchrotron radiation XAFS measurements were performed. As shown 
in Fig. 3a,b, the Sn quantum sheets confined in graphene exhibited a strong FT peak of $\mathrm{Sn}-\mathrm{Sn}$ metallic bonding at ca. $2.77 \AA$, while they did not possess the peaks for $\mathrm{Sn}^{4+}-\mathrm{O}$ at ca. $1.45 \AA$ and $\mathrm{Sn}-\mathrm{O}-\mathrm{Sn}$ at above $3 \AA$, which undoubtedly confirmed that the $\mathrm{Sn}$ quantum sheets were well protected from oxidation through spatially confining in the graphene. In addition, their Sn K-edges $k^{3} \chi(k)$ oscillation curves in Fig. 3a show obvious differences relative to the $15 \mathrm{~nm} \mathrm{Sn}$ nanoparticles and bulk counterpart (Supplementary Figs 1-3), which suggested their distinct local atomic arrangement further verified by their R-space curves in Fig. 3b. According to the high-quality extended XAFS spectra, least-squares fittings were further conducted and the obtained quantitative results are shown in Table 1. For the Sn quantum sheets confined in graphene, the coordination numbers of $\mathrm{Sn}-\mathrm{Sn}$ reduced from 2 and 4 to 1.4 and 2.7, while their disorder degrees increased significantly as compared with the $15 \mathrm{~nm} \mathrm{Sn}$ nanoparticles and bulk counterpart. This suggested that the reduced size resulted in many dangling bonds as well as a noticeable distortion on the surface of Sn quantum sheets, which would contribute to their high catalytic activity along with excellent stability $7,13,14$. Altogether, both the HRTEM and XAFS results disclosed that the synthetic Sn quantum sheets were free of oxidation under the effective umbrella of graphene.

Electroreduction of $\mathrm{CO}_{2}$ into hydrocarbon fuels. Benefiting from the lowered coordination number, obvious structure distortion as well as surface conductive graphene, the Sn quantum sheets confined in graphene were expected to give substantially promoted $\mathrm{CO}_{2}$ electroreduction performances in comparison with the Sn nanoparticles as well as the bulk counterpart. To verify the higher activity of the unique sandwich-like $2 \mathrm{D}$ structure, $\mathrm{CO}_{2}$ reduction activities for the $\mathrm{Sn}$ quantum sheets confined in graphene, $15 \mathrm{~nm} \mathrm{Sn}$ nanoparticles mixed with graphene, $15 \mathrm{~nm}$ Sn nanoparticles and bulk $\mathrm{Sn}$ were determined in $\mathrm{CO}_{2}$-saturated $0.1 \mathrm{M} \mathrm{NaHCO} 3$ solution. As revealed by linear sweep voltammetry (LSV) in Fig. $4 \mathrm{a}$, the $\mathrm{Sn}$ quantum sheets confined in graphene exhibited the highest catalytic activity among the above four samples. For instance, the Sn quantum sheets confined in graphene displayed a current density of $21.1 \mathrm{~mA} \mathrm{~cm}^{-2}$ at $-1.8 \mathrm{~V}$ versus SCE, which was roughly $2,2.5$ and 13 times larger than that of the $15 \mathrm{~nm}$ Sn nanoparticles mixed with graphene, $15 \mathrm{~nm}$ Sn nanoparticles and bulk Sn, respectively. In addition, the $\mathrm{Sn}$ quantum sheets confined in graphene exhibited an onset potential of $-0.85 \mathrm{~V}$ versus SCE, which was much positive relative to other three samples (Fig. 4a). This strongly demonstrated the simple addition of graphene could only slightly mend the catalytic activity, while the formation of unique sandwich construction can help to remarkably promote the activity.

To analyse the electroreduction products, stepped-potential electrolyses at each given potential for $4 \mathrm{~h}$ were performed to periodically quantify the liquid products by ${ }^{1} \mathrm{H}$ nuclear magnetic resonance spectroscopy and the gas products by an Agilent Technologies 7890B gas chromatograph ${ }^{2}$. As revealed by the faradaic efficiency for formate production in Fig. $4 \mathrm{~b}$, one can clearly observe that the Sn quantum sheets confined in graphene behaved obviously higher faradaic efficiency relative to other three samples in the whole applied potentials. At the potential of $-1.8 \mathrm{~V}$ versus SCE, the $\mathrm{Sn}$ quantum sheets
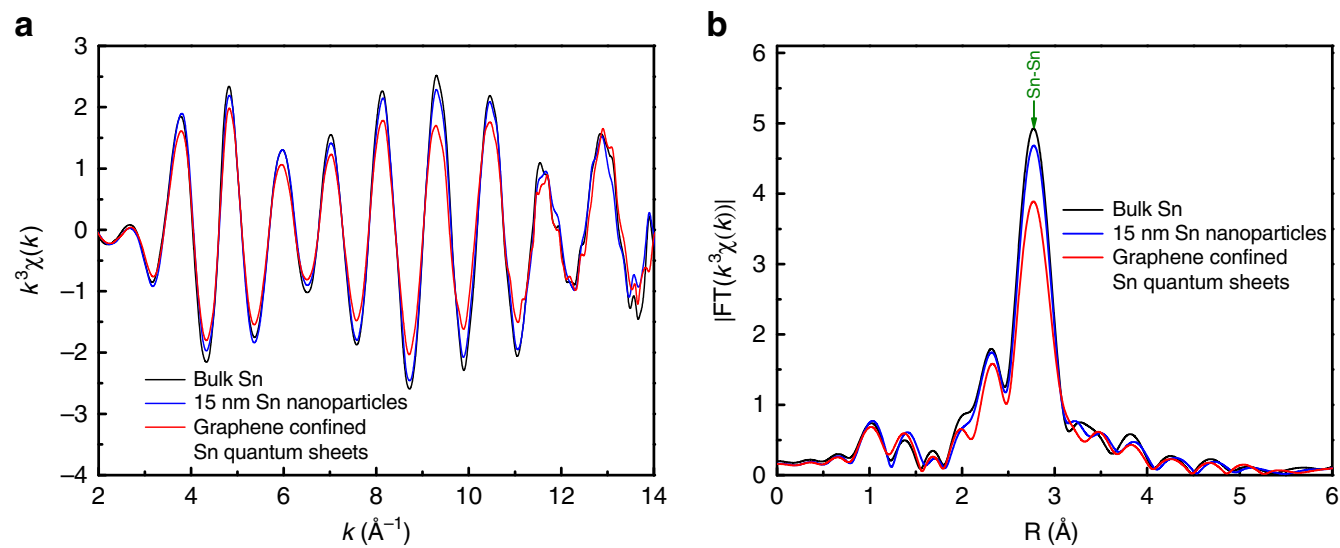

Figure 3 | Synchrotron radiation XAFS measurements. (a) Sn K-edge extended XAFS oscillation function $k^{3} \chi(k)$, (b) the corresponding Fourier transforms $\mathrm{FT}\left(k^{3} \chi(k)\right)$ for the graphene confined $\mathrm{Sn}$ quantum sheets, $15 \mathrm{~nm} \mathrm{Sn}$ nanoparticles and bulk $\mathrm{Sn}$, respectively.

Table 1 | EXAFS curve-fitting results.

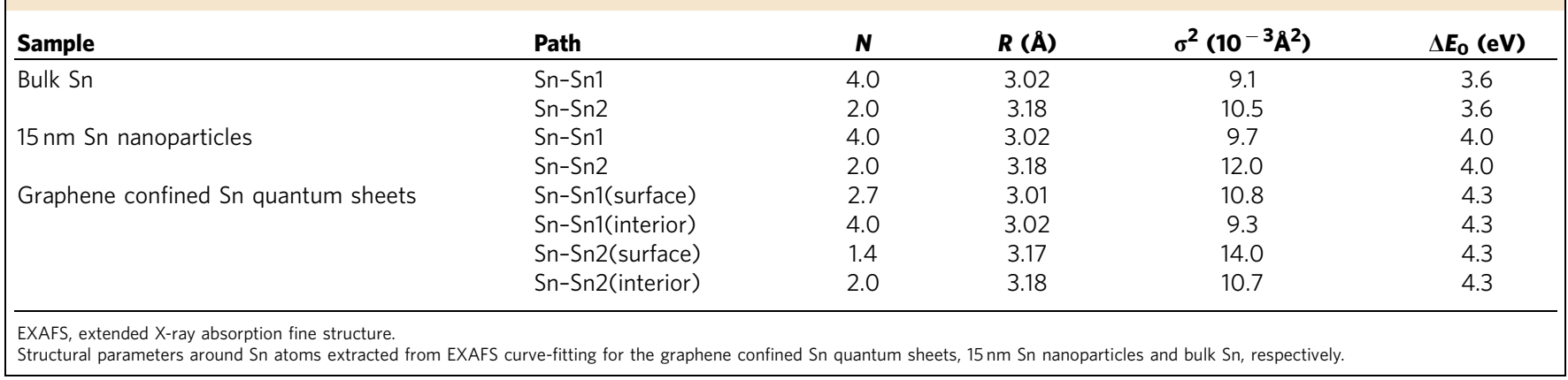


a

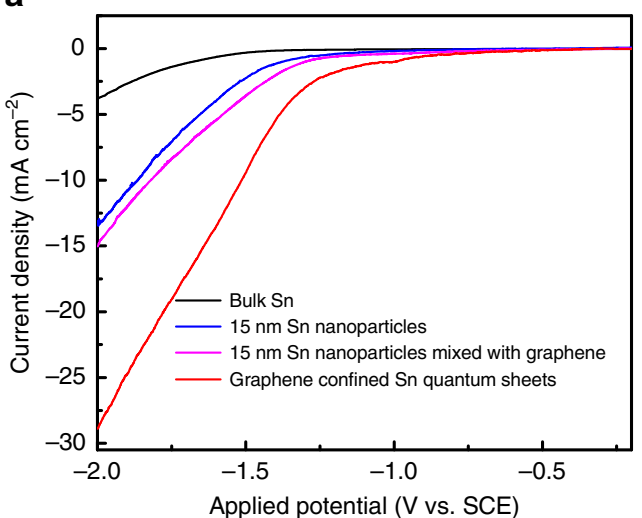

C

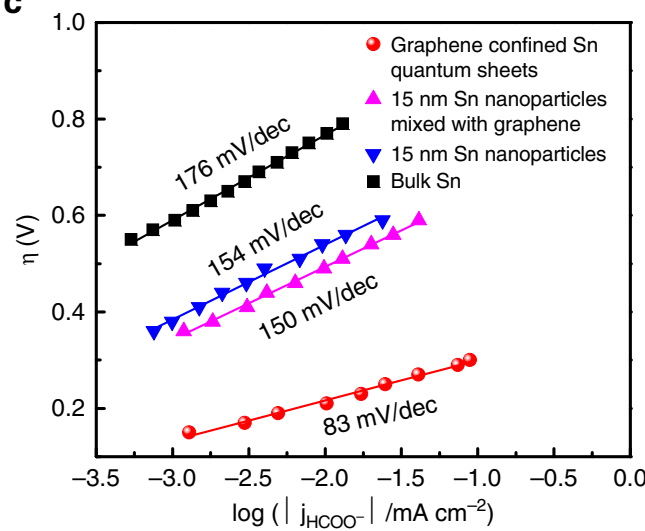

b

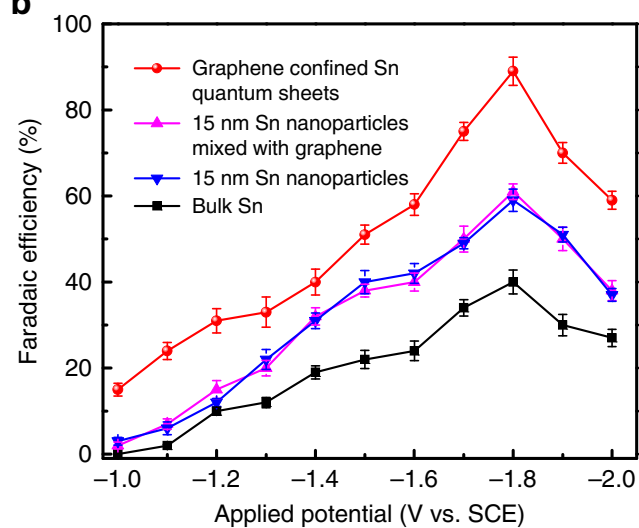

d

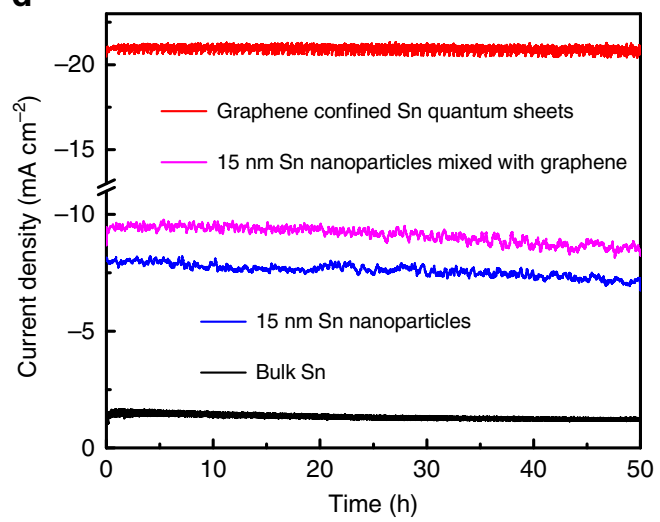

Figure $4 \mid \mathbf{C O}_{\mathbf{2}}$ electroreduction performances. (a) Linear sweep voltammetric curves in the $\mathrm{CO}_{2}$-saturated $0.1 \mathrm{M} \mathrm{NaHCO}$ aqueous solution, (b) Faradaic efficiencies for formate at each applied potentials for $4 \mathrm{~h}$, (c) Tafel plots for producing formate, (d) Chrono-Amperometry results at the potentials of $-1.8 \mathrm{~V}$ versus SCE for the Sn quantum sheets confined in graphene, $15 \mathrm{~nm}$ Sn nanoparticles mixed with graphene, $15 \mathrm{~nm}$ Sn nanoparticles and bulk Sn. The error bars in $\mathbf{b}$ represent the standard deviations of five independent measurements of the same sample.

confined in graphene attained a maximum faradaic efficiency of $89 \%$, which was roughly $1.45,1.5$ and 2 times higher than that of the $15 \mathrm{~nm}$ Sn nanoparticles mixed with graphene, $15 \mathrm{~nm} \mathrm{Sn}$ nanoparticles and bulk $\mathrm{Sn}$, denoting the superior activity of their unique structure. In addition, the faradaic efficiency for gas products in Supplementary Fig. 4 reveal that the main gas products are the $\mathrm{H}_{2}$, while $\mathrm{CO}$ accounted for the remaining products. Of note, the above four samples achieved the maximum formate faradaic efficiency at the similar potentials, which could be ascribed to the mass transport limitation of $\mathrm{CO}_{2}$ as well as the competitive $\mathrm{H}_{2}$ evolution reaction ${ }^{8,18}$. To obtain further insights into these electrocatalysts, their Tafel plots were investigated and presented in Fig. 4c. The resulting Tafel slope of the Sn quantum sheets confined in graphene was $83 \mathrm{mV} \mathrm{dec}^{-1}$, much smaller than that of the $15 \mathrm{~nm} \mathrm{Sn}$ nanoparticles mixed with graphene, $15 \mathrm{~nm}$ Sn nanoparticles and bulk Sn, respectively. The smaller Tafel slope of the Sn quantum sheets confined in graphene is very advantageous for practical applications, since it will lead to a much faster increment of $\mathrm{CO}_{2}$ reduction rate with increasing overpotential ${ }^{10}$. Besides the activity of $\mathrm{CO}_{2}$ electroreduction, electrochemical stability is another significant criterion to evaluate an advanced electrocatalyst. Hence, continuous $\mathrm{CO}_{2}$ reduction was performed at $-1.8 \mathrm{~V}$ versus SCE for $50 \mathrm{~h}$ in order to probe the durability of the above electrocatalysts. The Sn quantum sheets confined in graphene did not show any obvious decay in the current densities, while their Faradaic efficiency for producing formate was always larger than $85 \%$ during the long test period of $50 \mathrm{~h}$, indicative of their very favourable stability
(Fig. 4d). Contrastingly, all the $15 \mathrm{~nm}$ Sn nanoparticles mixed with graphene, $15 \mathrm{~nm}$ Sn nanoparticles and bulk Sn exhibited relatively poor long-term stability. Therefore, the $\mathrm{Sn}$ quantum sheets confined in graphene have sufficient potential as a promising catalyst for electrocatalytic $\mathrm{CO}_{2}$ conversion with a superior formate production rate as well as a prolonged stability.

\section{Discussion}

With regard to the notable enhancement of catalytic activity for the Sn quantum sheets confined in graphene, the increase in electrochemical active surface area (ECSA) may be one of the main contributors since larger ECSA could afford more catalytically active sites ${ }^{2}$. According to the measured double-layer capacitance in Fig. 5a, the ECSA for the Sn quantum sheets confined in graphene was estimated to be roughly $1.5,2$ and 28 times higher than that of the $15 \mathrm{~nm}$ Sn nanoparticles mixed with graphene, $15 \mathrm{~nm}$ Sn nanoparticles and bulk Sn. The increased ECSA for the Sn quantum sheets confined in graphene suggested their remarkably larger amount of active sites, which endowed them with increased $\mathrm{CO}_{2}$ adsorption capacity, verified by the corresponding $\mathrm{CO}_{2}$ adsorption isotherms in Fig. 5b. For instance, the amount of $\mathrm{CO}_{2}$ adsorption capacity for the Sn quantum sheets confined in graphene could reach $26.1 \mathrm{mg} \mathrm{g}^{-1}$ at $1 \mathrm{~atm}$, which is roughly $1.75,2$ and 9 times higher than that of the $15 \mathrm{~nm} \mathrm{Sn}$ nanoparticles mixed with graphene, $15 \mathrm{~nm}$ Sn nanoparticles and bulk counterpart. Compared with the $15 \mathrm{~nm}$ Sn nanoparticles, the $15 \mathrm{~nm}$ Sn nanoparticles mixed with 
a

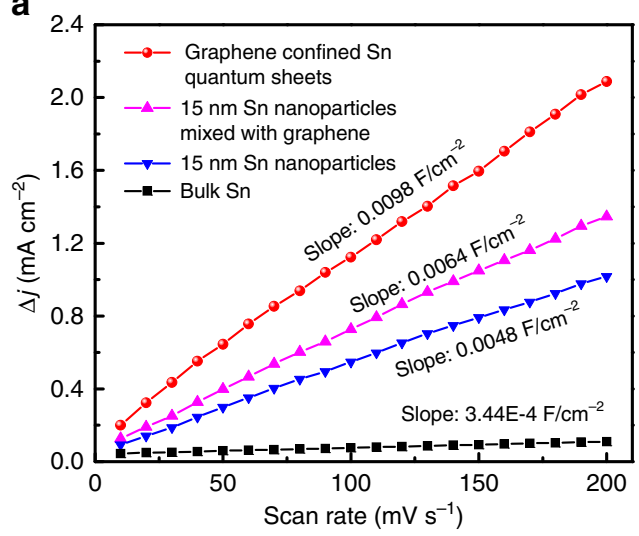

C

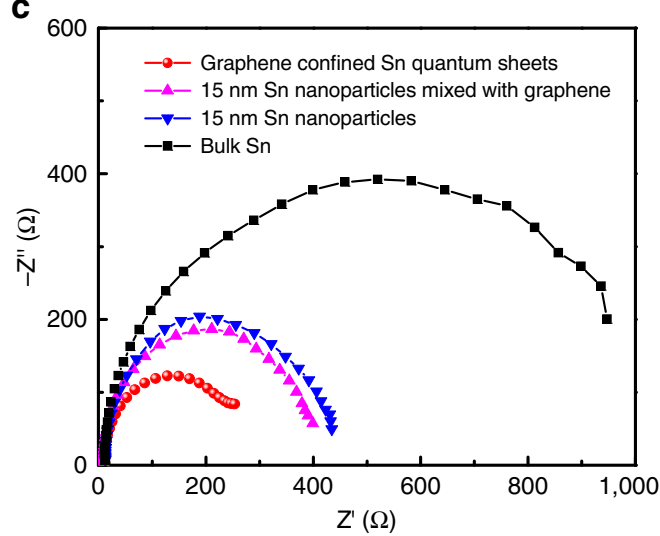

b

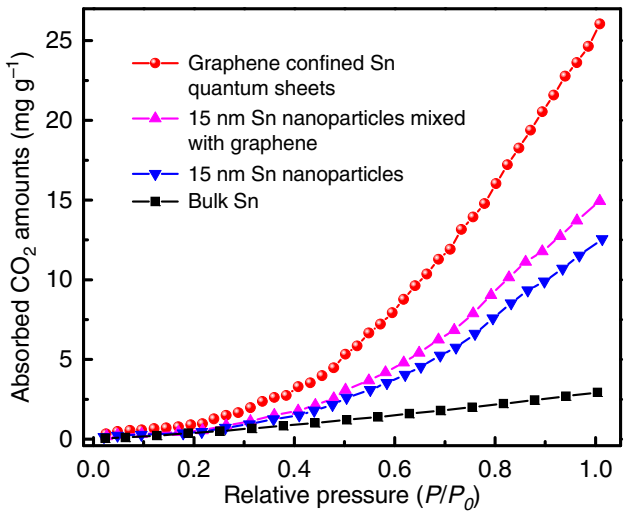

d

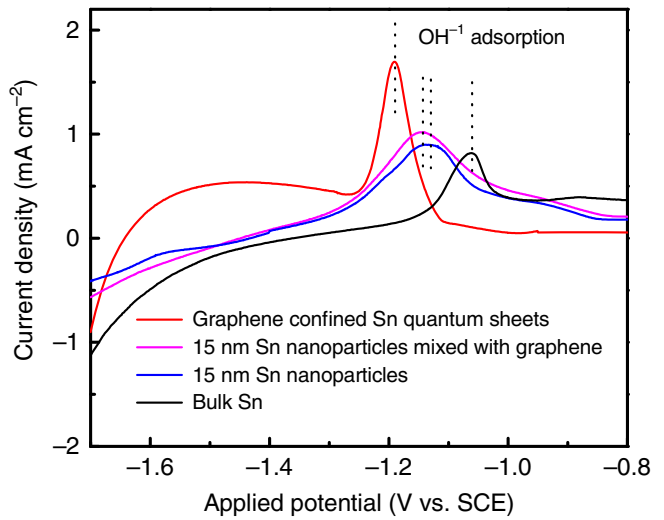

Figure 5 | Advantages of the Sn quantum sheets confined in graphene. (a) Charging current density differences plotted against scan rates; (b) $\mathrm{CO}_{2}$ adsorption isotherms; (c) Nyquist plots; (d) single oxidative LSV scans in $\mathrm{N}_{2}$-saturated 0.1 M NaOH for the Sn quantum sheets confined in graphene, $15 \mathrm{~nm}$ Sn nanoparticles mixed with graphene, $15 \mathrm{~nm}$ Sn nanoparticles and bulk Sn.

graphene possessed a slightly increased $\mathrm{CO}_{2}$ adsorption amount, which suggested that the graphene played a limited role in increasing the $\mathrm{CO}_{2}$ adsorption. Thus, the 28 times higher ECSA for the Sn quantum sheets confined in graphene, largely coming from the high surface area graphene, only led to ca. 9 times higher $\mathrm{CO}_{2}$ adsorption capacity relative to bulk $\mathrm{Sn}$, and hence only resulted in 13 times higher current density. Moreover, the lowered $\mathrm{Sn}$-Sn coordination number for the Sn quantum sheets confined in graphene, revealed by XAFS fitting results in Fig. 3, further indicated their higher intrinsic catalytic activity, which could be further demonstrated by their Tafel slope lowered from 176 to $83 \mathrm{mV} \mathrm{dec}^{-1}$ relative to bulk $\mathrm{Sn}$ in Fig. 4c. Moreover, the presence of highly-conductive graphene could enhance the overall electronic conductivity, which was confirmed by their lowest interfacial charge-transfer resistance in Fig. $5 c$, hence ensuring fast electron transfer to $\mathrm{CO}_{2}$ for forming $\mathrm{CO}_{2}^{-}$radiacal anion intermediate. Of note, the first electron transfer step of $\mathrm{CO}_{2}+\mathrm{e} \rightarrow \mathrm{CO}_{2}^{-}$is generally regarded as the rate-determining step ${ }^{1,2,4-6}$, in which stabilization of the $\mathrm{CO}_{2}^{-}$intermediate plays a fundamental role in $\mathrm{CO}_{2}$ reduction into formate. To testify the binding affinity of $\mathrm{CO}_{2}^{-}$on the four samples, adsorption of $\mathrm{OH}^{-}$as a surrogate for $\mathrm{CO}_{2}^{-}$was conducted by oxidative LSV scans under a $\mathrm{N}_{2}$-bubbled $0.1 \mathrm{M}$ $\mathrm{NaOH}$ electrolyte. The results in Fig. $5 \mathrm{~d}$ revealed that the potential for surface $\mathrm{OH}^{-}$adsorption on the Sn quantum sheets confined in graphene was $0.05,0.06$ and $0.13 \mathrm{~V}$ lower than that of the $15 \mathrm{~nm}$ Sn nanoparticles mixed with graphene, $15 \mathrm{~nm} \mathrm{Sn}$ nanoparticles and bulk Sn. This illustrated the Sn quantum sheets confined in graphene possessed stronger adsorption affinity of $\mathrm{OH}^{-}$, and hence they could efficiently stabilize the $\mathrm{CO}_{2}^{-}$intermediate ${ }^{8,19}$, finally facilitating formate production. Therefore, the above results strongly demonstrated that the simple addition of graphene could only slight improve the $\mathrm{CO}_{2}$ reduction performances, while the graphene interlayer confined Sn quantum sheets could fully optimize the crucial processes during $\mathrm{CO}_{2}$ electroreduction into formate.

In conclusion, we first put forward an ideal material model of ultrathin metal layers confined in graphene, in which the protection of graphene could avoid the oxidation of the highly reactive metals in air. As an example, highly reactive metallic Sn quantum sheets, confined in few-layered graphene, are first successfully synthesized through a spatially confined reduction strategy. Synchrotron radiation XAFS results disclose that $\mathrm{Sn}-\mathrm{Sn}$ coordination numbers for the $\mathrm{Sn}$ quantum sheets confined in graphene reduce from 2 and 4 to 1.4 and 2.7 compared with that of bulk counterpart, which implies the former's higher intrinsic catalytic activity, further demonstrated by their lowered Tafel slope from 176 to $83 \mathrm{mV} \mathrm{dec}^{-1}$. The remarkably increased ECSA for the Sn quantum sheets confined in graphene could afford larger amount of active sites to efficiently adsorb $\mathrm{CO}_{2}$, further confirmed by their 1.75, 2 and 9 times higher $\mathrm{CO}_{2}$ adsorption capacity compared with the $15 \mathrm{~nm}$ Sn nanoparticles mixed with graphene, $15 \mathrm{~nm} \mathrm{Sn} \mathrm{nanoparticles} \mathrm{and} \mathrm{bulk} \mathrm{Sn.} \mathrm{The}$ lowest interfacial charge-transfer resistance, benefiting from the highly-conductive graphene, facilitates the rate-limiting electron transfer from $\mathrm{CO}_{2}$ to $\mathrm{CO}_{2}^{-}$intermediate. In addition, compared to the $15 \mathrm{~nm}$ Sn nanoparticles mixed with graphene, $15 \mathrm{~nm} \mathrm{Sn}$ nanoparticles and bulk $\mathrm{Sn}$, the $0.05,0.06$ and $0.13 \mathrm{~V}$ lowered 
potential of $\mathrm{OH}^{-}$adsorption indicate that the Sn quantum sheets confined in graphene possess the strongest adsorption affinity of $\mathrm{CO}_{2}^{-}$intermediate, and hence they efficiently stabilize the $\mathrm{CO}_{2}^{-}$intermediate, thus definitely lowering the overall reaction barrier. As an outcome, the Sn quantum sheets confined in graphene display a current density of $21.1 \mathrm{~mA} \mathrm{~cm}^{-2}$ at $-1.8 \mathrm{~V}$ versus SCE, which is roughly $2,2.5$ and 13 times larger than that of the $15 \mathrm{~nm} \mathrm{Sn}$ nanoparticles mixed with graphene, $15 \mathrm{~nm} \mathrm{Sn}$ nanoparticles and bulk Sn. Also, the formate Faradaic efficiency for Sn quantum sheets confined in graphene is always larger than $85 \%$ during the long test period of $50 \mathrm{~h}$. Thus, the present work demonstrates that the unique structure of highly reactive metal quantum sheets confined in graphene could fully optimize $\mathrm{CO}_{2}$ electroreduction performances.

\section{Methods}

Synthesis of Sn quantum sheets confined in grapheme. In a typical synthesis, $10 \mathrm{mg}$ ultrathin $\mathrm{SnO}_{2}$ layers, synthesized according to our previous work (Angew. Chem. Int. Ed. 2013, 52, 10,569), were dispersed in $40 \mathrm{ml} 0.03 \mathrm{M}$ aqueous glucose solution under mild stirring for $30 \mathrm{~min}$. Then the above mixture was transferred into a $50 \mathrm{ml}$ Teflon-lined autoclave, sealed and heated at $180^{\circ} \mathrm{C}$ for $10 \mathrm{~h}$. After the initial hydrothermal method, the ultrathin $\mathrm{SnO}_{2}$ layers were homogenerously encapsulated in the carbon layer. Then, the carbon-coated ultrathin $\mathrm{SnO}_{2}$ layers were initially annealed at $500{ }^{\circ} \mathrm{C}$ for $2 \mathrm{~h}$, and then immediately annealed at $1,000^{\circ} \mathrm{C}$ for $5 \mathrm{~min}$, and hence cooled down rapidly (within $1 \mathrm{~min}$ ) to room temperature under the protection of argon atmosphere. During this temperature-programed process, the amorphous carbon turned into graphene, while the ultrathin $\mathrm{SnO}_{2}$ layers simultaneously reduced into monodispersed Sn quantum sheets in the confined space of graphene, thus forming a sandwich-like structure. By contrast, for the synthesis of $15 \mathrm{~nm}$ Sn nanoparticles mixed with graphene, the $15 \mathrm{~nm} \mathrm{Sn}$ nanoparticles, synthesized according to the ref. of Nanotechnology, 2011, 22, 22,5701, were physically mixed with the commercial graphene under mild stirring for $1 \mathrm{~h}$. Then, the $15 \mathrm{~nm}$ Sn nanoparticles could be dispersed on the surface of graphene.

Synthesis of bulk Sn. In a typical synthesis, $50 \mathrm{mg}$ ultrathin $\mathrm{SnO}_{2}$ layers, synthesized according to ref. Angew. Chem. Int. Ed. 2013, 52, 10,569, were annealed at $500^{\circ} \mathrm{C}$ for $5 \mathrm{~h}$ in $\mathrm{H}_{2}$ atmosphere and then cooled down to room temperature. The obtained powders were collected for further characterization.

Characterization. TEM images and high-resolution TEM image were performed by using a JEOL-2010 TEM with an acceleration voltage of $200 \mathrm{kV}$. The Sn K-edge XAFS was measured at the Shanghai Synchrotron Radiation Factory and the National Synchrotron Radiation Laboratory, China. Thermal gravimetric analysis (TGA) of the as-synthesized samples were carried out on a Shimadzu TA-50 thermal analyser at a heating rate of $10^{\circ} \mathrm{C} \mathrm{min}^{-1}$ from room temperature to $700^{\circ} \mathrm{C}$ in air. The liquid products were quantified by nuclear magnetic resonance (Bruker AVANCE AV III 400) spectroscopy. $\mathrm{CO}_{2}$ adsorption isotherms measurements for all the synthetic samples were carried out by using an automatic microporous physical and chemical gas adsorption analyser (ASAP $2020 \mathrm{M}+\mathrm{C}$ )

\section{Electrochemical measurements. Electrochemical measurements were} implemented in a three-electrode system at an electrochemical station (CHI660E). The working electrode was glassy carbon electrode. The platinum gauze and the saturated calomel electrode (SCE) reference electrode served as counter and the reference electrodes, respectively. In a typical prepared procedure of the working electrode, $3 \mu \mathrm{l}$ of the homogeneous ink, which was prepared by dispersing $5 \mathrm{mg}$ sample and $40 \mu \mathrm{l}$ Nafion solution $(5 \mathrm{wt} \%)$ in $1 \mathrm{ml}$ water-ethanol solution with volume ratio of 3:1, was loaded onto a glassy carbon electrode with $3 \mathrm{~mm}$ diameter. For $\mathrm{CO}_{2}$ reduction experiments, LSV with a scan rate of $20 \mathrm{mV} \mathrm{s}^{-1}$ was carried out in $\mathrm{CO}_{2}$-saturated $0.1 \mathrm{M} \mathrm{NaHCO}_{3}$ solution $(60 \mathrm{ml})$ (The $\mathrm{NaHCO}_{3}$ electrolyte was purged with $\mathrm{CO}_{2}$ for 30 min prior to the measurement.). ECSA was determined by measuring the capacitive current associated with double-layer charging from the scan-rate dependence of CVs. The $C_{\mathrm{dl}}$ was estimated by plotting the $\Delta j\left(j_{\mathrm{a}}-j_{\mathrm{c}}\right)$ at $-0.75 \mathrm{~V}$ versus SCE against the scan rate, where the $\Delta j$ could be acquired by Cyclic voltammetry measurement under the potential windows of $-0.8 \sim-0.7 \mathrm{~V}$ versus SCE $\left(0.1 \mathrm{M} \mathrm{NaHCO}_{3}\right.$ solution)

EXAFS experimental details. Sample of $20 \mathrm{mg}$ was homogeneously mixed with $100 \mathrm{mg}$ graphite and hence pressed into circular pellets with a diameter of $10 \mathrm{~mm}$ for further extended X-ray absorption fine structure (EXAFS) measurement under ambient conditions. Then, the XAFS measurements were performed at BL14W1 station in Shanghai Synchrotron Radiation Facility, China.
The storage rings of SSRF were operated at $3.5 \mathrm{GeV}$ with the maximum current of $210 \mathrm{~mA}$. Si(311) double-crystal monochromator crystals were used to monochromatize the X-ray beam. The energy resolution at Sn K-edge was about $2 \mathrm{eV}$. XAFS data were collected in transmission in the energy range from -200 below to $1,000 \mathrm{eV}$ above the $\mathrm{Sn} \mathrm{K}$-edge. The detuning was done by $30 \%$ to remove harmonics.

Data availability. The authors declare that the data supporting the findings of this study are available within the article and its Supplementary Information files and from the corresponding author upon reasonable request.

\section{References}

1. Li, C. W., Ciston, J. \& Kanan, M. W. Electroreduction of carbon monoxide to liquid fuel on oxide-derived nanocrystalline copper. Nature 508, 504-507 (2014).

2. Gao, S. et al. Partially oxidized atomic cobalt layers for carbon dioxide electroreduction to liquid fuel. Nature 529, 68-71 (2016).

3. Rosen, B. A. et al. Ionic liquid-mediated selective conversion of $\mathrm{CO}_{2}$ to $\mathrm{CO}$ at low overpotentials. Science 334, 643-644 (2011).

4. Chen, Y. H., Li, C. W. \& Kanan, M. W. Aqueous $\mathrm{CO}_{2}$ reduction at very low overpotential on oxide-derived Au nanoparticles. J. Am. Chem. Soc. 134, 19969-19972 (2012)

5. Chen, Y. H. \& Kanan., M. W. Tin oxide dependence of the $\mathrm{CO}_{2}$ reduction efficiency on tin electrodes and enhanced activity for tin/tin oxide thin-film catalysts. J. Am. Chem. Soc. 134, 1986-1989 (2012).

6. Li, C. W. \& Kanan, M. W. $\mathrm{CO}_{2}$ reduction at low overpotential on $\mathrm{Cu}$ electrodes resulting from the reduction of thick $\mathrm{Cu}_{2} \mathrm{O}$ Films. J. Am. Chem. Soc 134, 7231-7234 (2012)

7. Sun, Y. F., Gao, S. \& Xie, Y. Atomically-thick two-dimensional crystals: electronic structure regulation and energy device construction. Chem. Soc. Rev. 43, 530-546 (2014).

8. Zhang, S., Kang, P. \& Meyer, T. J. Nanostructured Tin Catalysts for Selective Electrochemical Reduction of Carbon Dioxide to Formate. J. Am. Chem. Soc. 136, 1734-1737 (2014).

9. Chen, S. et al. N-doped graphene film-confined nickel nanoparticles as a highly efficient three-dimensional oxygen evolution electrocatalyst. Energy Environ. Sci 6, 3693-3699 (2013).

10. Gao, S. et al. Ultrathin $\mathrm{Co}_{3} \mathrm{O}_{4}$ layers realizing optimized $\mathrm{CO}_{2}$ electroreduction to formate. Angew. Chem. Int. Ed. 55, 698-702 (2016).

11. Liang, L. et al. Single unit cell bismuth tungstate layers realizing robust solar $\mathrm{CO}_{2}$ reduction to methanol. Angew. Chem. Int. Ed. 54, 13971-13974 (2015).

12. Deng, J. et al. Enhanced electron penetration through an ultrathin graphene layer for highly efficient catalysis of the hydrogen evolution reaction. Angew. Chem. Int. Ed. 54, 2100-2104 (2015).

13. Sun, Y. F. et al. Fabrication of flexible and freestanding zinc chalcogenide single layers. Nat. Commun 3, 1057 (2012).

14. Sun, Y. F. et al. Pits confined in ultrathin cerium(IV) oxide for studying catalytic centers in carbon monoxide oxidation. Nat. Commun. 4, 2899 (2013).

15. Medina-Ramos, J. et al. Efficient conversion of $\mathrm{CO}_{2}$ to $\mathrm{CO}$ using tin and other inexpensive and easily prepared post-transition metal catalysts. J. Am. Chem. Soc. 137, 5021-5027 (2015).

16. Sun, Y. F. et al. Atomically thin tin dioxide sheets for efficient catalytic oxidation of carbon monoxide. Angew. Chem. Int. Ed. 52, 10569-10572 (2013)

17. Guo, X. K. et al. Sandwich-like $\mathrm{LiFePO}_{4} /$ graphene hybrid nanosheets: in situ catalytic graphitization and their high-rate performance for lithium ion batteries. J. Mater. Chem. A 1, 11534-11538 (2013)

18. Humar, B. et al. Renewable and metal-free carbon nanofibre catalysts for carbon dioxide reduction. Nat. Commun. 4, 2819 (2013).

19. Won, D. H. et al. Rational design of a hierarchical tin dendrite electrode for efficient electrochemical reduction of $\mathrm{CO}_{2}$. Chemsuschem 8, 3092-3098 (2015)

\section{Acknowledgements}

This work was financially supported by National Nature Science Foundation (21422107, $21331005,91422303,11321503,11422547)$, the National Basic Research Program of China (2015CB932302), Youth Innovation Promotion Association of CAS (CX2340000100), the Fundamental Research Funds for the Central Universities No. WK2340000063, WK2340000073 and Scientific Research Grant of Hefei Science Center of CAS (2016HSC-IU002, 2015HSC-UE006, 2015HSC-UP015).

\section{Author contributions}

Y.X., Y.S., T.Y., F.L. and W.L. conceived the idea and co-wrote the paper. F.L., J.X., K.L. and L.L.carried out the sample synthesis, characterization and $\mathrm{CO}_{2}$ reduction meansurement. Y.S., F.L. and B.P. discussed the 
catalytic process. W.L., T.Y. and S.W. performed and analysed the XAFS data. All the authors discussed the results, commented on and revised the manuscript.

\section{Additional information}

Supplementary Information accompanies this paper at http://www.nature.com/ naturecommunications

Competing financial interests: The authors declare no competing financial interests.

Reprints and permission information is available online at http://npg.nature.com/ reprintsandpermissions/
How to cite this article: Lei, F. et al. Metallic tin quantum sheets confined in graphene toward high-efficiency carbon dioxide electroreduction. Nat. Commun. 7:12697 doi: 10.1038/ncomms12697 (2016).

(c) This work is licensed under a Creative Commons Attribution 4.0 International License. The images or other third party material in this article are included in the article's Creative Commons license, unless indicated otherwise in the credit line; if the material is not included under the Creative Commons license, users will need to obtain permission from the license holder to reproduce the material. To view a copy of this license, visit http://creativecommons.org/licenses/by/4.0/

(C) The Author(s) 2016 